\title{
Hernia diafragmática secundaria a trauma: presentación tardía
}

\author{
Marcelo A. Beltrán S. ${ }^{1,2}$, Juan David Oyarzún S. ${ }^{1,2}$, Daniela Castro G. ${ }^{2}$, \\ Paola Andrade L. ${ }^{2}$ y Carolina Fernández R. ${ }^{2}$
}

\section{Complicated diaphragmatic hernia: late outcome of trauma}

Introduction: Late presentation of traumatic diaphragmatic hernia constitutes a true surgical emergency. The purposes of this study were to describe the diagnostic characteristics, treatment and outcomes of late presentation diaphragmatic hernias and to identify a cutoff point from the onset of symptoms to necrosis development. Patients and Methods: A retrospective series of cases constituted by all patients subjected to emergency diaphragmatic hernia repair form 2006 to 2016 was studied. Categorical and continuous variables were measured and analyzed with descriptive statistics. Receiver Operating Characteristics (ROC) curves at 6 and 12 hours from the onset of symptoms were used to determine the cutoff point for organ resection. Once stablished the cutoff point, sensitivity, specificity, positive and negative predictive values and prevalence were calculated. Results: Clinical presentation, diagnostic study and surgical treatment were similar to what has been already described. The cutoff point was defined at 12 hours from the onset of symptoms with $80 \%$ sensitivity and $83 \%$ sensibility. Conclusions: The diagnosis and treatment of these cases should be carried on before the first 6 hours after the onset of symptoms. Even though the universe of this study was small, we may suggest that after 12 hours form the onset of symptoms, the implicated organs would be found necrotic requiring surgical resection.

Key words: diaphrafgmatic hernia; gastric volvulus; gastric necrosis; late complications of thoracic trauma; late complications of abdominal trauma.

\section{Resumen}

Introducción: La hernia diafragmática complicada de presentación tardía constituye una urgencia quirúrgica. Los objetivos del presente estudio son la descripción de las características del diagnóstico de las hernias diafragmáticas de presentación tardía, tratamiento, resultados y la identificación del punto de corte entre el inicio de los síntomas y el desarrollo de necrosis. Pacientes y Métodos: Estudio retrospectivo de una serie de casos constituida por todos los pacientes operados por hernia diafragmática complicada de presentación tardía entre los años 2006 y 2016. Se midieron variables categóricas y continuas que se presentan con estadística descriptiva. Se utilizaron curvas Receiver Operating Characteristics (ROC) a las 6 y $12 \mathrm{~h}$ desde el inicio de los síntomas, para determinar el punto de corte del tiempo de presentación clínica en pacientes sometidos a resección de algún órgano. Una vez establecido el punto de corte se calcularon la sensibilidad; especificidad; las razones de verosimilitud positiva y negativa; los valores predictivos positivo y negativo; y la prevalencia. Resultados: La presentación clínica, estudio, diagnóstico y tratamiento fue similar a lo descrito en la literatura quirúrgica. Se estableció el punto de corte a las $12 \mathrm{~h}$ con sensibilidad de $80 \%$ y especificidad de $83 \%$. Conclusiones: El diagnóstico y tratamiento de estos pacientes debería ser llevado a cabo antes de las $6 \mathrm{~h}$ desde el inicio de los síntomas. Aun cuando el universo descrito es reducido, se sugiere que después de las $12 \mathrm{~h}$ desde el momento de la estrangulación, los órganos comprometidos se encontrarán necróticos requiriendo resección quirúrgica.

Palabras clave: hernia diafragmática; vólvulo gástrico; necrosis gástrica; complicaciones tardías del trauma torácico; complicaciones tardías del trauma abdominal. 


\section{Introducción}

Las hernias diafragmáticas se clasifican en congénitas y adquiridas por traumatismos. La hernia diafragmática traumática se diagnostica durante una de las siguientes tres fases: fase aguda que se presenta inmediatamente después del trauma; la fase de intervalo o de latencia entre el trauma y la complicación, que se asocia a síntomas transitorios y ocasionales causados por la herniación de distintos órganos abdominales y reducción espontánea de las vísceras; y la fase de las complicaciones cuando la hernia se complica con obstrucción y estrangulación de los órganos contenidos en el saco herniario ${ }^{1}$. Las hernias diafragmáticas traumáticas son indirectas secundarias a trauma cerrado y directas secundarias a trauma penetrante de la región torácica o abdominal ${ }^{2}$. La incidencia de ruptura diafragmática después de un trauma penetrante del tórax inferior o hemiabdomen superior es de $15 \%^{3}$; en trauma torácico o abdominal cerrado la incidencia varía entre $0,8 \%$ y $6 \%$ de los casos $^{1-9}$. Aproximadamente $30 \%$ de las hernias diafragmáticas traumáticas se presentan en forma tardía, con un período de intervalo entre la injuria y la presentación que varía entre $24 \mathrm{~h}$ y 50 años $^{9-11}$. La hernia diafragmática complicada de presentación tardía constituye una urgencia quirúrgica debido a la isquemia, eventual necrosis y gangrena de los órganos herniados ${ }^{1-13}$. Los objetivos del presente estudio son la descripción de las características del diagnóstico de las hernias diafragmáticas de presentación tardía, tratamiento, resultados y la identificación del punto de corte entre el inicio de los síntomas y el desarrollo de necrosis de los órganos comprometidos.

\section{Pacientes y Métodos}

Estudio retrospectivo de una serie de casos constituida por todos los pacientes operados por hernia diafragmática complicada de presentación tardía secundaria a herida penetrante de tórax o abdomen entre los años 2006 y 2016 en el Servicio de Emergencias del Hospital San Juan de Dios de La Serena.

\section{Criterios de exclusión}

Se excluyeron todos los pacientes operados por hernias diafragmáticas secundarias a trauma abierto operados en forma electiva. También se excluyeron todos los pacientes operados por urgencia debido a trauma torácico o abdominal, abierto o cerrado, en los que se encontró una hernia diafragmática como parte de sus lesiones. Finalmente, se excluyeron todos los pacientes que en el momento de su presentación con complicaciones de la hernia fueron menores de 15 años.

\section{Variables de estudio}

Se estudiaron variables continuas y categóricas que incluyeron: edad del paciente, lugar de la herida, procedimiento o cirugía primaria posinjuria, tiempo de hospitalización posinjuria, tiempo de latencia entre la injuria y la complicación de la hernia, tiempo de presentación clínica entre el inicio de los síntomas y el diagnóstico de hernia diafragmática complicada, características de presentación clínica, estudio radiológico, tamaño del defecto del diafragma, contenido de la hernia, cirugía a la que se sometió el paciente, complicaciones posoperatorias y tiempo de hospitalización poscirugía.

\section{Definiciones}

Se definió como hernia diafragmática complicada de presentación tardía a aquella hernia que se presentó después de una lesión penetrante de tórax o abdomen, que fue evaluada o tratada requiriendo o no cirugía y hospitalización sin el diagnóstico de hernia diafragmática secundaria a trauma, siendo el paciente dado de alta y reingresando 7 o más días después del alta con complicaciones secundarias a la hernia. Se definió como "latencia" al período de tiempo transcurrido entre la lesión primaria y la presentación del paciente con una hernia diafragmática complicada. Las lesiones del diafragma que se encontraron en la cirugía fueron clasificadas de acuerdo al comité Organ Injury Scaling de la American Association for the Surgery of Trauma ${ }^{14}$ (Tabla 1). Las complicaciones posoperatorias se clasificaron de acuerdo a la clasificación de Clavien-Dindo ${ }^{15}$.

\section{Medida de resultado}

La medida de resultado principal fue la de establecer un punto de corte para el desarrollo de necrosis de los órganos comprometidos en la hernia complicada.

\section{Estadística}

Se midieron variables categóricas y continuas, las que se presentan con estadística descriptiva y medidas de dispersión generales. Se utilizaron curvas Receiver Operating Characteristics (ROC) a las 6 y $12 \mathrm{~h}$ desde el inicio de los síntomas, para determinar el punto de corte del tiempo de presentación clínica entre pacientes sometidos a resección de algún órgano herniado y los que no requirieron ninguna resección. Una vez establecido el punto de corte se calcularon la sensibilidad; especificidad; las 
razones de verosimilitud positiva y negativa (RVP y RVN); los valores predictivos positivo y negativo (VPP y VPN); y la prevalencia. Para este cálculo se dividió arbitrariamente la serie de casos de acuerdo al tiempo de presentación clínica en pacientes que se presentaron antes de las $6 \mathrm{~h}$, entre las 7 y $11 \mathrm{~h}$ y 12 o más horas después del inicio de los síntomas. La base de datos se construyó y analizó con el software estadístico SSPS 11.0.

\section{Resultados}

Un total de 9 pacientes fueron incluidos en el estudio. Sobre la base de los criterios de exclusión no se excluyeron pacientes. En todos los pacientes, la herida inicial que lesionó el diafragma fue producida por arma blanca. Todos los pacientes fueron de sexo masculino. El promedio de edad fue 24,1 años (18 a 35). La mayoría de los pacientes sufrieron heridas en la base del hemitórax izquierdo o en el hemiabdomen superior izquierdo; en un caso se describen múltiples heridas en tórax y abdomen y en otro caso la herida fue epigástrica. La mayoría de los pacientes ( 5 casos) fueron tratados con una pleurostomía izquierda. Dos pacientes fueron sometidos a laparotomía exploradora sin encontrar la lesión diafragmática y otros dos pacientes no fueron sometidos a ninguna intervención y no se hospitalizaron. El promedio de hospitalización en los restantes 7 casos fue 2,8 días (Tabla 2). El promedio del período de latencia fue de 449 días (7 a 1.080). Todos los pacientes relataron un inicio súbito de la sintomatología. El promedio de la duración del cuadro clínico desde el inició de los síntomas hasta el diagnóstico fue de 18,6 h (4 a 72). La clínica en todos los casos fue inespecífica y se caracterizó por dolor torácico o abdominal superior intenso de ini-
Tabla 1. Clasificación de las lesiones diafragmáticas

\begin{tabular}{|cl|}
\hline Grados & Descripción \\
\hline I & Contusión \\
\hline II & Laceración igual o menor a $2 \mathrm{~cm}$ \\
\hline III & Laceración de 2 a $10 \mathrm{~cm}$ \\
IV & Laceración mayor a $10 \mathrm{~cm}$ con pérdida de tejido menor a $25 \mathrm{~cm}^{2}$ \\
V & Laceración mayor a $10 \mathrm{~cm}$ con pérdida de tejido mayor a $25 \mathrm{~cm}^{2}$ \\
\hline
\end{tabular}

cio súbito, taquicardia, taquipnea y una auscultación pulmonar alterada con ausencia de ruidos ventilatorios. La mayoría de los pacientes se estudiaron con una radiografía de tórax y en un caso se tomó una radiografía contrastada de estómago con contraste oral hidrosoluble (Figura 1). En 4 casos también
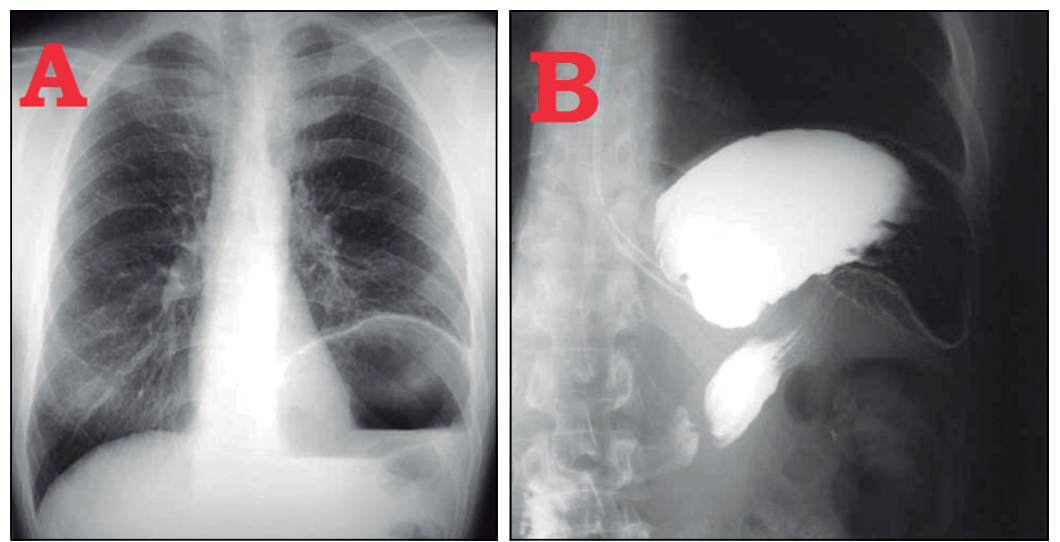

Figura 1. Radiografía simple y radiografía contrastada de tórax evidenciando el estómago intratorácico. A. Radiografía de tórax antero-posterior de pie. Se observa claramente el estómago dentro del hemitórax izquierdo en un paciente de 23 años que se presentó dentro de las primeras 6 horas de sufrir la complicación aguda de una hernia diafragmática. B. Radiografía contrastada de tórax en el mismo paciente, la cual identifica el estómago en su posición intratorácica delineado por el contraste.

Tabla 2. Características de la lesión original

\begin{tabular}{|cllc|}
\hline Edad & Mecanismo de la lesión original & Cirugía primaria y hallazgos & Hospitalización \\
18 & Herida en hemiabdomen superior izquierdo & Laparotomía: Hemoperitoneo sin lesiones de órganos & 3 días \\
19 & Herida en hemitórax izquierdo & Pleurostomía: Neumotórax + Hemotórax & No días \\
\hline 19 & Heridas múltiples en tórax y abdomen & No se operó & días \\
21 & Herida en hemitórax izquierdo & Pleurostomía: Neumotórax & 5 días \\
\hline 23 & Herida epigástrica & Laparotomía: Hemoperitoneo y perforación gástrica & 3 días \\
\hline 24 & Herida en hemitórax izquierdo & Pleurostomía: Hemotórax & días \\
\hline 28 & Herida en hemitórax izquierdo & Pleurostomía: Hemotórax & No \\
\hline 30 & Herida en hemiabdomen superior izquierdo & No se operó & 3 días \\
\hline
\end{tabular}


se tomó una tomografía axial computarizada (TC) de tórax y abdomen (Figuras 2 y 3). La mayoría de los estudios radiológicos permitieron establecer el diagnóstico de una hernia diafragmática complicada (Tabla 3). El promedio del tamaño del defecto encontrado en la cirugía fue de 4,6 cm (3 a 7) lo que corresponde a lesiones diafragmáticas Grado III. En todos los pacientes la hernia contenía epiplón mayor, en 6 casos entre otros órganos contenía además el estómago. En un caso se encontró el bazo y el ángulo esplénico del colon; en otro paciente contenía íleon, bazo y colon; y en un último caso colon, bazo y estómago. En 4 pacientes el estómago se encontraba necrótico por lo que fueron sometidos a gastrectomía total; en los otros 5 casos no se resecaron otros órganos y la reducción del contenido hacia la cavidad abdominal fue suficiente. Todos los defectos se repararon con puntos separados de sutura absorbible. Se presentaron complicaciones en un paciente que requirió una toracotomía para tratar un empiema pleural organizado (Clavien-Dindo IIIb) (Tabla 4). De acuerdo con el análisis de la curva

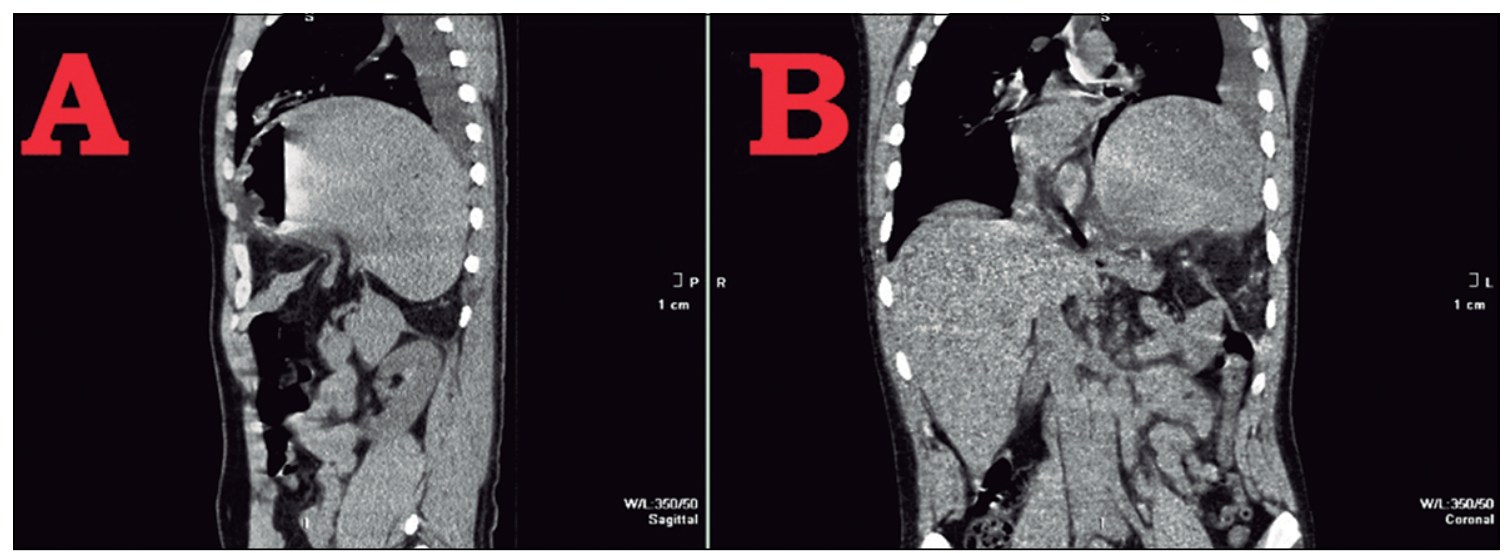

Figura 2. Reconstrucción sagital y coronal de una tomografía de abdomen y tórax. Reconstrucción tomográfica en un paciente de 21 años que se presentó con un vólvulo gástrico con necrosis de todo el estómago, 18 meses después de sufrir un trauma penetrante de tórax por arma blanca. A. Corte sagital que demuestra el estómago dentro del tórax, se puede apreciar el defecto del diafragma que medía aproximadamente $4 \mathrm{~cm}$. B. Corte coronal en el mismo paciente en el cual se observa el estómago intratorácico.
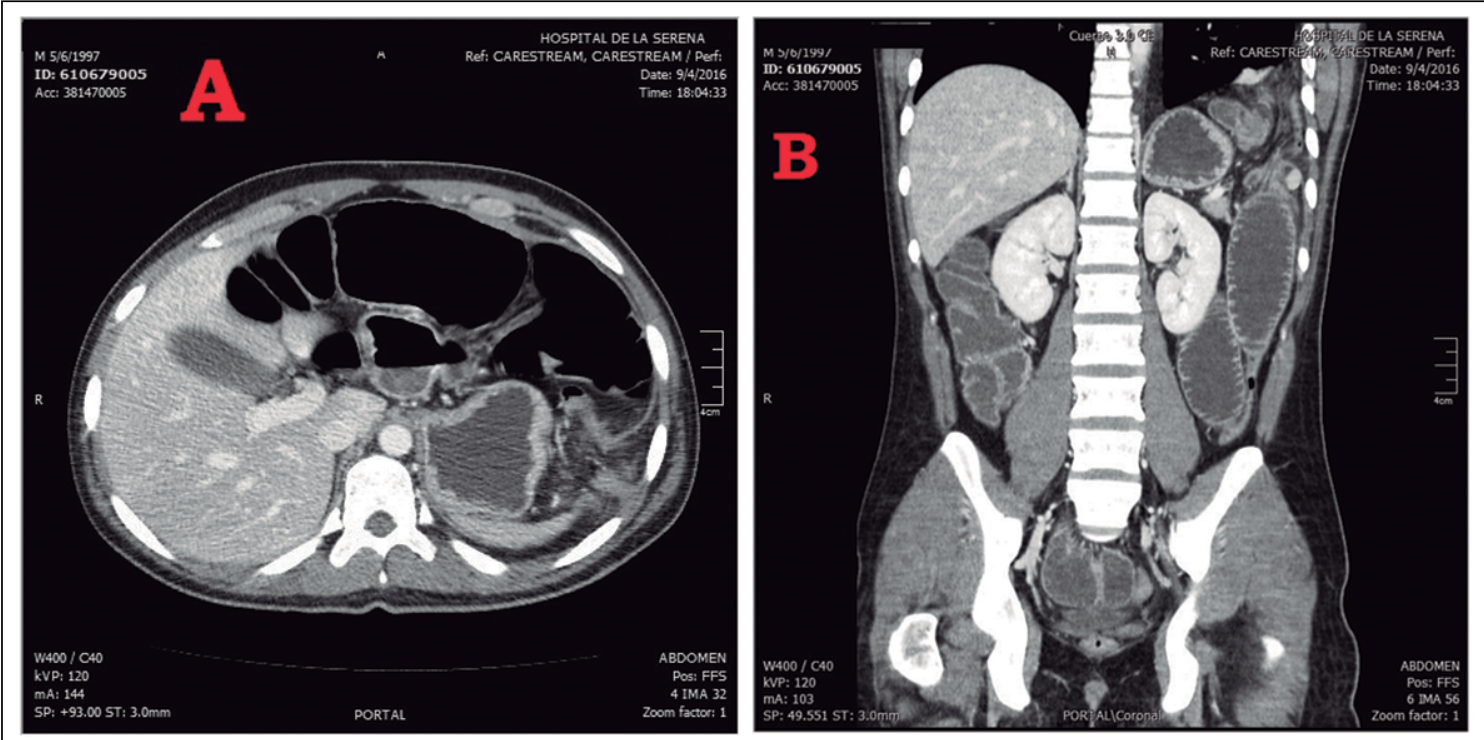

Figura 3. Cortes transversal y coronal en hernia diafragmática. A. Paciente de 19 años con hernia diafragmática izquierda posterior que contenía estómago, bazo, ángulo esplénico del colon y epiplón mayor que requirieron una gastrectomía total y reducción sin resección del bazo y ángulo esplénico del colon. B. Corte coronal en el mismo paciente en el que se observa el defecto y el contenido de la hernia. 
Tabla 3. Características de la presentación clínica tardía por la hernia diafragmática complicada

\begin{tabular}{|c|c|c|c|c|c|}
\hline Edad & Latencia & Presentación & Clínica & Radiología & Hallazgos radiológicos \\
\hline 18 & $1 \mathrm{mes}$ & 4 horas & $\begin{array}{l}\text { Dolor abdominal. Vómito. Fiebre. } \\
\text { Taquicardia. Taquipnea. Examen pulmonar } \\
\text { alterado }\end{array}$ & $\begin{array}{l}\text { Rx Tórax } \\
\text { TAC Tórax y } \\
\text { Abdomen }\end{array}$ & $\begin{array}{l}\text { Hernia diafragmática que contiene } \\
\text { colon con nivel hidroaéreo }\end{array}$ \\
\hline 19 & 2 semanas & 8 horas & $\begin{array}{l}\text { Dolor torácico. Taquipnea. Taquicardia. } \\
\text { Examen pulmonar alterado. Vómito }\end{array}$ & Rx Tórax & $\begin{array}{l}\text { Imagen en tórax sospechosa de un } \\
\text { quiste hidatídico complicado }\end{array}$ \\
\hline 21 & 18 meses & 24 horas & $\begin{array}{l}\text { Dolor abdominal. Dolor torácico. Taquipnea. } \\
\text { Taquicardia. Examen pulmonar alterado }\end{array}$ & $\begin{array}{l}\text { Rx Tórax } \\
\text { TAC Tórax y } \\
\text { Abdomen }\end{array}$ & $\begin{array}{l}\text { Hernia diafragmática que contiene } \\
\text { estómago }\end{array}$ \\
\hline 23 & 15 meses & 6 horas & $\begin{array}{l}\text { Dolor abdominal. Vómito. Fiebre. } \\
\text { Taquicardia. Taquipnea. Examen pulmonar } \\
\text { alterado }\end{array}$ & $\begin{array}{l}\text { Rx Tórax* } \\
\text { TAC Tórax y } \\
\text { Abdomen }\end{array}$ & $\begin{array}{l}\text { Hernia diafragmática que contiene } \\
\text { estómago }\end{array}$ \\
\hline 30 & 1 semana & 72 horas & $\begin{array}{l}\text { Dolor torácico. Vómito. Taquipnea. } \\
\text { Taquicardia. }\end{array}$ & Rx Tórax & $\begin{array}{l}\text { Hernia diafragmática que contiene } \\
\text { estómago }\end{array}$ \\
\hline 35 & 27 meses & 32 horas & $\begin{array}{l}\text { Dolor torácico. Taquipnea. Taquicardia. } \\
\text { Examen pulmonar alterado }\end{array}$ & Rx Tórax & $\begin{array}{l}\text { Hernia diafragmática que contiene } \\
\text { estómago }\end{array}$ \\
\hline
\end{tabular}

Rx: Radiografía-TAC: Tomografía Axial Computarizada. RxTórax*: En este caso se utilizó contraste oral (bario diluido) para delinear el contenido de la hernia.

Tabla 4. Hallazgos quirúrgicos, cirugía y complicaciones posoperatorias

\begin{tabular}{|c|c|c|c|c|c|}
\hline Edad & Tamaño del defecto $(\mathrm{cm})$ & Contenido & Cirugía & Complicaciones & Clavien \\
\hline 18 & 5 & $\begin{array}{l}\text { Íleon } \\
\text { Colon } \\
\text { Epiplón mayor } \\
\text { Bazo }\end{array}$ & $\begin{array}{l}\text { Reducción de contenido } \\
\text { Sutura del defecto }\end{array}$ & No & - \\
\hline 19 & 5 & $\begin{array}{l}\text { Estómago } \\
\text { Epiplón mayor } \\
\text { Bazo } \\
\text { Colon }\end{array}$ & $\begin{array}{l}\text { Reducción del contenido } \\
\text { Gastrectomía total } \\
\text { Sutura del defecto }\end{array}$ & $\begin{array}{l}\text { Empiema pleural tratado } \\
\text { con toracotomía }\end{array}$ & IIIb \\
\hline 19 & 3 & $\begin{array}{l}\text { Estómago } \\
\text { Epiplón mayor }\end{array}$ & $\begin{array}{l}\text { Reducción del contenido } \\
\text { Sutura del defecto }\end{array}$ & No & - \\
\hline 21 & 4 & $\begin{array}{l}\text { Estómago } \\
\text { Epiplón mayor }\end{array}$ & $\begin{array}{l}\text { Gastrectomía total } \\
\text { Sutura del defecto }\end{array}$ & No & - \\
\hline 23 & 6 & $\begin{array}{l}\text { Estómago } \\
\text { Epiplón mayor }\end{array}$ & $\begin{array}{l}\text { Reducción del contenido } \\
\text { Sutura del defecto }\end{array}$ & No & - \\
\hline 24 & 5 & $\begin{array}{l}\text { Colon } \\
\text { Estómago } \\
\text { Bazo } \\
\text { Epiplón mayor }\end{array}$ & $\begin{array}{l}\text { Reducción del contenido } \\
\text { Sutura del defecto }\end{array}$ & No & - \\
\hline 28 & 4 & $\begin{array}{l}\text { Colon } \\
\text { Epiplón mayor }\end{array}$ & $\begin{array}{l}\text { Reducción del contenido } \\
\text { Sutura del defecto }\end{array}$ & No & - \\
\hline 30 & 3 & $\begin{array}{l}\text { Estómago } \\
\text { Epiplón mayor }\end{array}$ & $\begin{array}{l}\text { Gastrectomía total } \\
\text { Sutura del defecto }\end{array}$ & No & - \\
\hline 35 & 3 & $\begin{array}{l}\text { Estómago } \\
\text { Epiplón mayor }\end{array}$ & $\begin{array}{l}\text { Gastrectomía total } \\
\text { Sutura del defecto }\end{array}$ & No & - \\
\hline
\end{tabular}




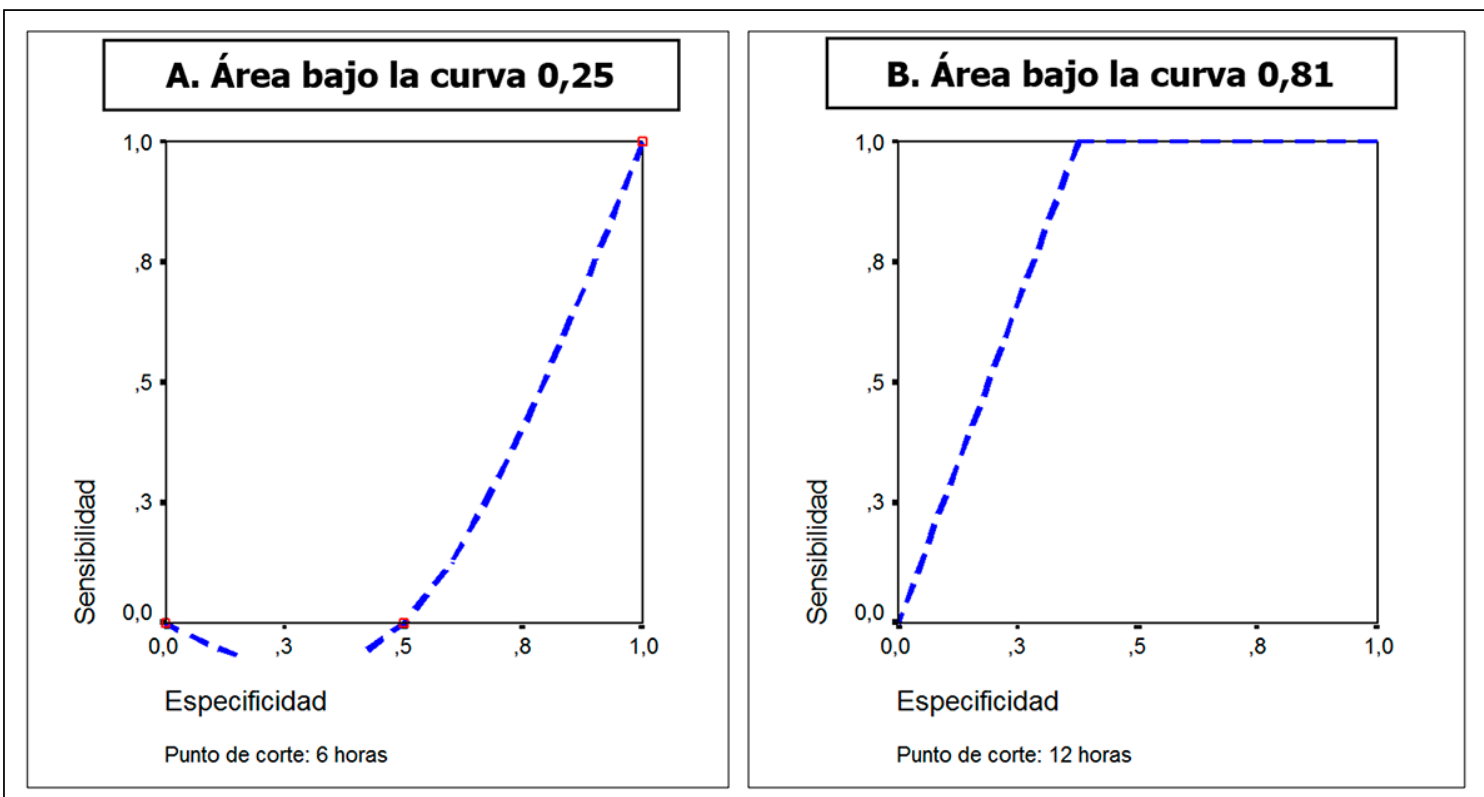

Figura 4. Curvas ROC a las 6 y $12 \mathrm{~h}$ del inicio de la sintomatología. A. Curva ROC que muestra un área bajo la curva menor a 0,50 utilizando como punto de corte las $6 \mathrm{~h}$ desde el inicio del cuadro clínico. Este hecho sugiere que los pacientes que se presentan con $6 \mathrm{~h}$ o menos tiempo de evolución presentan poco riesgo de sufrir la resección de los órganos comprometidos en la hernia. B. Curva ROC que muestra un área bajo la curva mayor a 0,50 utilizando el punto de corte a las $12 \mathrm{~h}$ desde el inicio del cuadro clínico. Este hecho sugiere que los pacientes que se presentan con $12 \mathrm{~h}$ o más de evolución tienen un elevado riesgo de sufrir la resección de los órganos comprometidos en la hernia.

ROC, es posible observar que aquellos pacientes con $6 \mathrm{~h}$ o menos de evolución del cuadro clínico desde el inicio de los síntomas hasta el diagnóstico tienen pocas probabilidades de sufrir isquemia, necrosis, gangrena y resección de los órganos herniados (Figura 4A). Por otro lado, aquellos pacientes que se presentaron después de 12 o más horas desde el inicio de la sintomatología, tienen un elevado riesgo de sufrir complicaciones isquémicas que lleven a la resección de los órganos herniados (Figura 4B).

Tabla 5. Resultados de las pruebas diagnósticas

\begin{tabular}{|lcc|}
\hline Prueba & Resultado & $\begin{array}{c}\text { Intervalos de confianza: } \\
\mathbf{9 5 \%}\end{array}$ \\
\hline Sensibilidad & $80 \%$ & $56-104$ \\
\hline Especificidad & $83 \%$ & $61-105$ \\
\hline Razón de verosimilitud positiva & 4,80 & - \\
\hline Razón de verosimilitud negativa & 0,24 & - \\
\hline Valor predictivo positivo & $80 \%$ & $56-104$ \\
Valor predictivo negativo & $83 \%$ & $61-105$ \\
\hline Prevalencia & $45 \%$ & $16-75$ \\
\hline
\end{tabular}

El análisis con pruebas diagnósticas muestra que el punto de corte de las $12 \mathrm{~h}$ tiene una sensibilidad de $80 \%$ y una especificidad de $83 \%$ constituyéndose en un parámetro importante a considerar cuando se presentan estos pacientes (Tabla 5).

\section{Discusión}

En el paciente con múltiples injurias, la lesión más obvia y de mayor gravedad es rápidamente identificada y tratada. Otras lesiones menos aparentes, como una laceración del diafragma, pasan desapercibidas y permiten la herniación del contenido abdominal hacia el tórax en forma precoz o tardía resultando en una hernia diafragmática, la cual puede permanecer asintomática en forma indefinida o sufrir complicaciones después de un período de tiempo desde el trauma inicial ${ }^{1-10}$. Este período de latencia varía considerablemente y en nuestros pacientes se prolongó hasta por 3 años; durante esta fase los pacientes presentan una sintomatología inespecífica con molestias abdominales leves hasta severo dolor en hemiabdomen superior debido a la incarceración intermitente de las vísceras herniadas y sintomatología respiratoria o cardiológica debi- 
Tabla 6. Fases de la evolución clínica de la hernia diafragmática

\begin{tabular}{|c|c|c|}
\hline Fase aguda & Fase de intervalo & Fase de complicaciones \\
\hline $\begin{array}{l}\text { Desde la lesión inicial hasta la recuperación } \\
\text { aparente }\end{array}$ & $\begin{array}{l}\text { Periodo en el cual los órganos intra- } \\
\text { abdominales migran hacia el tórax }\end{array}$ & $\begin{array}{l}\text { Se produce meses o años después de la lesión } \\
\text { cuando los órganos herniados se complican }\end{array}$ \\
\hline $\begin{array}{l}\text { - Dolor abdominal } \\
\text { - Otras lesiones asociadas } \\
\text { - Inestabilidad hemodinámica } \\
\text { - Dificultad respiratoria } \\
\text { - Disminución de la ventilación del lado afec- } \\
\text { tado } \\
\text { - Auscultación de ruidos intestinales intra- } \\
\text { torácicos } \\
\text { - Anomalías en la radiografía de tórax } \\
\text { - Lesión identificada durante la cirugía de } \\
\text { urgencia }\end{array}$ & $\begin{array}{l}\text { - Dolor abdominal superior } \\
\text { - Disnea o cianosis } \\
\text { - Taquicardia } \\
\text { - Dolor retroesternal referido hacia el } \\
\text { hombro } \\
\text { - Disnea exacerbada en posición supina } \\
\text { - Matidez a la percusión y disminución } \\
\text { de los ruidos pulmonares } \\
\text { - Auscultación de ruidos intestinales } \\
\text { intratorácicos } \\
\text { - Anomalías en la radiografía de tórax }\end{array}$ & $\begin{array}{l}\text { - Náusea y vómito } \\
\text { - Síntomas de obstrucción intestinal, isquemia } \\
\text { o hemorragia digestiva } \\
\text { - Dificultad respiratoria crónica } \\
\text { - Desviación del mediastino } \\
\text { - Triada de Borchardt: } \\
\text { Dolor abdominal superior, flatulencia y } \\
\text { dificultad para instalar una sonda naso- } \\
\text { gástrica } \\
\text { - Auscultación de ruidos intestinales intra- } \\
\text { torácicos }\end{array}$ \\
\hline
\end{tabular}

do al espacio que ocupan en el tórax los órganos herniados (Tabla 6) ${ }^{2,3}$. La presentación tardía de la hernia diafragmática se debe también en otros casos, a que un número significativo de pacientes con lesiones abdominales no requieren cirugía y a que la mayoría de los pacientes con lesiones penetrantes de tórax requieren solo drenaje pleural y observación, razones por las cuales las pequeñas lesiones del diafragma pasan desapercibidas ${ }^{3-10}$. En muchos casos, el origen de la hernia diafragmática es secundario a trauma abdominal o torácico cerrado asociado a múltiples lesiones como fracturas costales, hemotórax, neumotórax, trauma esplénico, trauma hepático, trauma intestinal, fracturas óseas, etc ${ }^{3-8}$. La ruptura tardía del músculo desvitalizado puede producirse varios días después del accidente ${ }^{3,12}$. Las heridas del diafragma raramente cicatrizan debido a que los bordes de la lesión se mantienen separados uno de otro por la presión intraabdominal positiva y por la interposición del omento mayor o algún otro órgano abdominal, que es con mayor frecuencia el estómago $^{1-3}$. Además, los movimientos respiratorios del diafragma y la presión intratorácica negativa gradualmente producen la herniación del contenido abdominal hacia la cavidad torácica.

Las tasas de morbilidad y mortalidad de la hernia diafragmática traumática dependen de la severidad de las lesiones asociadas y la demora en su diagnóstico ${ }^{7,8}$. Los órganos que se encuentran con mayor riesgo de sufrir complicaciones dentro de la hernia son el colon, estómago, bazo, epiplón mayor e intestinos ${ }^{6,11}$. El estómago es el órgano que más frecuentemente sufre herniación hacia el tórax ${ }^{3-13}$. Aun así, el vólvulo gástrico intratorácico asociado a necrosis gástrica es una de las complicaciones menos frecuentes de las hernias diafragmáticas de presentación tardía $(7 \% \text { de todos los casos })^{8}$. La causa de la necrosis del estómago es el vólvulo gástrico agudo. La necrosis y gangrena del estómago se deben al estrangulamiento del estómago dentro de la hernia, constituyendo una complicación severa con una mortalidad que alcanza al $33 \%$ de los ca$\operatorname{sos}^{1-6,10-13}$. Cuando el estómago necrótico se perfora puede presentarse como neumotórax, derrame pleural y sepsis ${ }^{3}$. En esta serie, todos los pacientes que requirieron la resección de un órgano, se presentaron con necrosis gástrica por vólvulo.

El diagnóstico de una hernia diafragmática complicada se apoya en los antecedentes del paciente y la severa sintomatología asociada ${ }^{2,3}$. La hernia diafragmática de presentación tardía, se presenta con complicaciones en alrededor del 80\% de los $\operatorname{casos}^{10}$. Así como en nuestros pacientes, la mayoría son de género masculino con un promedio de 35 años de edad que varía entre 15 y 80 años ${ }^{1-10}$. El síntoma más importante es el dolor torácico izquierdo anterior que suele ser de inicio súbito, pungitivo, constante, intenso, con irradiación hacia el brazo y hombro izquierdos y a la región epigástrica y que se exacerba con la inspiración profunda asociándose a vómitos explosivos ${ }^{1,3,5}$. Estos pacientes relatan una historia previa de dolores ocasionales en el hemitórax izquierdo asociados a disnea, tos y cuadros intermitentes de dispepsia y, ocasionalmente, obstrucción intestinal ${ }^{3,4,6,8,10}$. Cuando se presentan en los Servicios de Urgencias, se encuentran con compromiso respiratorio, disnea, taquipnea, taquicardia, fiebre $\mathrm{y}$, ocasionalmente, hipertensión $\operatorname{arterial}^{3,4,8}$. A la percusión se evidencia matidez en el hemitórax izquierdo y a la auscultación disminución o ausencia del murmullo vesicular y, ocasionalmente, ruidos peristálticos ${ }^{1-5}$. El abdomen puede 
encontrarse excavado en la región epigástrica, blando, y moderadamente sensible ${ }^{1-5}$. No se palpan masas intraabdominales ${ }^{3}$. El electrocardiograma es habitualmente normal ${ }^{1-4}$. Los pacientes de nuestra serie, presentaron estas características clínicas que se encuentran extensamente descritas en la literatura quirúrgica.

El estudio radiológico en estos casos habitualmente confirma el diagnóstico. La radiografía antero-posterior de tórax simple de pie permite rápidamente sospechar el diagnóstico de una hernia diafragmática complicada, el cual se confirma con una TC de tórax y abdomen ${ }^{16-21}$. Todos nuestros pacientes fueron estudiados con una radiografía de tórax y en 4 casos se realizaron TC de tórax y abdomen, esto se debe a la reciente incorporación de un tomógrafo en nuestra institución. En todos los casos los estudios radiológicos asociados al cuadro clínico y a los antecedentes de los pacientes, permitieron llegar al diagnóstico.

La exploración quirúrgica estableció la necesidad de resección del órgano comprometido en 4 casos debido a la necrosis o gangrena del mismo. Se ha demostrado que en el caso de la estrangulación de intestino o del estómago el flujo arterial se detiene a los 2 min desde el momento en el que se estrangula el órgano ${ }^{22}$. En la medida en la que el período de tiempo entre el momento de la estrangulación y el tratamiento se prolonga, se produce un fallo progresivo de la microcirculación ${ }^{23-25}$. Como consecuencia la isquemia continúa hasta que a las $24 \mathrm{~h}$ más del $50 \%$ de los órganos isquémicos se encuentran necróticos ${ }^{24-26}$. Este proceso que se inicia con la insuficiencia vascular, afecta inicialmente la mucosa y submucosa, en la medida que va progresando, compromete toda la pared del órgano produciéndose la necrosis del mismo lo cual constituye la fase final e irreversible ${ }^{25,26}$. En aquellos casos en los que el proceso continúa por falta de resolución, se llega al compromiso sistémico del paciente ${ }^{26}$. El análisis de nuestra pequeña serie de casos nos permite sugerir que en aquellos pacientes que se presentan 12 o más horas después del inicio de la sintomatología, el riesgo de sufrir necrosis y resección de los órganos comprometidos es elevado comparado con aquellos pacientes que se presentan antes de las $12 \mathrm{~h}$. Seguramente, un universo mayor de pacientes, nos permitiría establecer un punto de corte más específico para la ventana de tiempo entre la isquemia reversible y la necrosis irreversible.

\section{Conclusiones}

Las características clínicas y diagnósticas de las hernias diafragmáticas complicadas de presentación tardía, así como el tratamiento quirúrgico y sus resultados en esta serie, fueron similares a lo descrito en la literatura quirúrgica. El diagnóstico y tratamiento de estos pacientes debería ser llevado a cabo idealmente antes de las $6 \mathrm{~h}$ desde el inicio de los síntomas. Aun cuando el universo descrito es reducido, parece esperarse que después de las $12 \mathrm{~h}$ desde el momento de la estrangulación, los órganos comprometidos se encontrarán necróticos requiriendo resección quirúrgica.

\section{Responsabilidades éticas}

Protección de personas y animales. Los autores declaran que para esta investigación no se han realizado experimentos en seres humanos ni en animales.

Confidencialidad de los datos. Los autores declaran que han seguido los protocolos de su centro de trabajo sobre la publicación de datos de pacientes.

Derecho a la privacidad y consentimiento informado. Los autores declaran que en este artículo no aparecen datos de pacientes.

Financiamiento: Sin financiamiento.

Conflicto de Intereses: No hay.

\section{Referencias}

1. Kingsnorth AN, Gunning AJ. An unusual complication of traumatic diaphragmatic hernia. Thorax 1979;34:125-6.

2. Grimes OF. Traumatic injuries of the diaphragm. Am J Surg. 1974;128: 175-81.

3. Beltrán MA. Presentación tardía de hernia diafragmática traumática con necrosis gástrica: Revisión de la literatura científica. Rev Colomb Cir. 2013;28:5463.

4. Beltrán MA. Presentación tardía de hernia diafragmática con necrosis gástrica. Rev Hispanoamericana Hernia 2013;1:49-53.

5. Csapó G, Pátkay J. Stomach rupture complicating traumatic diaphragmatic hernia. Br Med J. 1969;2:312-3.

6. Ellis H. Diaphragmatic hernia - A diagnostic challenge. Postgrad Med J. 1986;62:325-7.

7. Christophi C. Diagnosis of traumatic diaphragmatic hernia: Analysis of 63 cases. World J Surg. 1983;7:277-80.

8. Özgüç H, Akköse Ş, Şen G, Bulut M, Kaya E. Factors affecting mortality and 
morbidity after traumatic diaphragmatic injury. Surg Today 2007;37:1042-6.

9. Rashid F, Chakrabarty MM, Singh

$\mathrm{R}$, Iftikhar SY. A review on delayed presentation of diaphragmatic rupture. World J Emerg Surg. 2009;4:32.

10. Tsuboi K, Omura N, Kashiwagi H, Kawasaki N, Suzuki Y, Yanaga K. Delayed traumatic diaphragmatic hernia after open splenectomy: Report of a case. Surg Today 2008;38:352-4.

11. Hegarty MM, Bryer JV, Angorn IB, Baker LW. Delayed presentation of traumatic diaphragmatic hernia. Ann Surg. 1978;188:229-33.

12. Yakaryilmaz F, Banli O, Altun H, Guliter S. Delayed presentation of post-traumatic diaphragmatic hernia with gastric volvulus: A case report. Turk J Trauma Emerg Surg. 2010;16:277-9.

13. Alper B, Vargun R, Kologlu MB, Fitoz S, Suskan E, Dindar H. Late presentation of a traumatic rupture of the diaphragm with gastric volvulus in a child: Report of a case. Surg Today 2007;37:874-7.

14. Moore EE, Moore FA. American Association for the Surgery of Trauma Organ Injury Scaling: 50th anniversary review article of the Journal of Trauma. J
Trauma 2010;69:1600-1.

15. Dindo D, Demartines N, Clavien PA. Classification of surgical complications: A new proposal with evaluation in a cohort of 6336 patients and results of a survey. Ann Surg. 2004;240:205-13.

16. Shiyoiti E, Gonçalves V, Benedito J, Hoelz de Oliveira R, Gomes NM, Pereira G. Plain chest radiographs for the diagnosis of post-traumatic diaphragmatic hernia. Rev Col Bras Cir. 2012;39:280-5.

17. Picetti E, Mergoni M. Traumatic diaphragmatic hernia. New Engl J Med. 2011;365:14.

18. Mirvis SE, Shanmuganagthan K. Imaging hemidiaphragmatic injury. Eur Radiol 2007;17:1411-21.

19. Santamarina MG, Rinaldi S, Baltazar AD, Volpacchio MM. Rol de la TC multicorte en las hernias diafragmáticas. Ensayo iconográfico. Rev Arg Radiol. 2009;73:421-32.

20. Bergin D, Ennis R, Keogh C, Fenlon HM, Murray JG. The "dependent viscera" sign in CT diagnosis of blunt traumatic diaphragmatic rupture. Am J Radiol. 2001;177:1137-40.

21. Llanos JC, Paredes NS, Schiappacasse
GF, Escalona AP, O’Brien A. Hernia diafragmática traumática complicada: Presentación como íleo. Rev Chil Radiol. 2005; 11:166-9.

22. Matsui A, Winer JH, Laurence RG, Frangioni JV. Predicting the survival of ischemic bowel in preclinical model systems using intraoperative near infrared fluorescence angiography. Br J Surg. 2011;98:1725-34.

23. Gorey TF. The recovery of the intestine after ischemic injury. Br J Surg. 1980;67:699-702.

24. Bralin RE, Orland PJ, Bibbo C, Reddell MT, Fedorciw MT, Gazi G, et al. Comparison of blood flow and myoelectric measurements in two chronic models of mesenteric ligation. Arch Surg. 1995;130:147-52.

25. Parks DA, Granger DN. Contributions of ischemia and reperfusion to mucosal lesion formation. Am J Physiol. 1986;250:G749-G753.

26. Peoc'h K, Nuzzo A, Guedj K, Paugam C, Corcos O. Diagnosis biomarkers in acute intestinal ischemic injury: So close, yet so far. Clin Chem Lab Med. 2017;DOI: 10.1515/cclm-2017-0291. [Epub ahead of print]. 